\title{
The Super-Connectivity of the Double Vertex Graph of Complete Bipartite Graphs
}

\author{
Gülnaz Boruzanlı Ekinci \\ Department of Mathematics, Faculty of Science, Ege University, İzmir, Turkey
}

\section{Article Info}

Keywords: Connectivity, Double vertex graph, Super connectivity, Token graph 2010 AMS: 05C40, 94C15, 05D05

Received: 28 July 2021

Accepted: 26 October 2021

Available online: 1 December 2021

\begin{abstract}
Let $G=(V, E)$ be a graph. The double vertex graph $F_{2}(G)$ of $G$ is the graph whose vertex set consists of all 2-subsets of $V(G)$ such that two vertices are adjacent in $F_{2}(G)$ if their symmetric difference is a pair of adjacent vertices in $G$. The super-connectivity of a connected graph is the minimum number of vertices whose removal results in a disconnected graph without an isolated vertex. In this paper, we determine the super-connectivity of the double vertex graph of the complete bipartite graph $K_{m, n}$ for $m \geq 4$ where $n \geq m+2$.
\end{abstract}

\section{Introduction}

Throughout this paper, let $G$ be a simple finite graph, where $V(G)$ and $E(G)$ denote the set of vertices and the set of edges, respectively. A set $S \subset V(G)$ is a vertex-cut of $G$, if $G-S$ is disconnected or has only one vertex. The neighbourhood of a vertex $v$ is the set $N_{G}(v)=\{u \in V(G): u v \in E(G)\}$. The degree of a vertex $v$, denoted by $\operatorname{deg}_{G}(v)$, is the cardinality of $N_{G}(v)$. Let $\delta(G)$ denote the minimum vertex degree in $G$. Two paths are internally disjoint if they have no common vertex except the end vertices. A set of paths is called internally disjoint if these paths are pairwise internally disjoint.

The double vertex graph $F_{2}(G)$ of $G$ is the graph whose vertex set consists of all the 2-subsets of $V(G)$ and two vertices are adjacent in $F_{2}(G)$ if their symmetric difference is a pair of adjacent vertices in $G$. That is, the vertices $\{u, v\}$ and $\{x, y\}$ of $F_{2}(G)$ are adjacent if and only if $|\{u, v\} \cap\{x, y\}|=1$ with $u=x$ and $v y \in E(G)$ (See Fig 1.1 for an example).

The notion of double vertex graph was introduced and studied by Alavi et al. [1]-[3]. The same concept was used by Rudolph to study the graph isomorphism problem under the name of symmetric power of a graph [4]. Later, Rudolph et al. [5] defined symmetric $k^{\text {th }}$ power of a graph $G$ as a generalization of symmetric power. In 2012, Fabila-Monroy et al. [6] introduced the notion of $k$-token graphs, which was a redefinition of symmetric $k^{\text {th }}$ powers of graphs. The $k$-token graph $F_{k}(G)$ of $G($ or, symmetric $k^{\text {th }}$ power of a graph $G$ ) is the graph whose vertices are all $k$-subsets of $V(G)$, where two vertices are adjacent if their symmetric difference is an edge in $E(G)$. Obviously, double vertex graphs correspond to 2-token graphs.

Note that if $G$ is a connected graph, then its double vertex graph is bipartite if and only if $G$ is bipartite. Also note that the degree of a vertex $\omega=\{x, y\}$ in $F_{2}(G)$ is given by

$$
\operatorname{deg}_{F_{2}(G)} \omega= \begin{cases}\operatorname{deg}_{G}(x)+\operatorname{deg}_{G}(y), & \text { if } x y \notin E(G), \\ \operatorname{deg}_{G}(x)+\operatorname{deg}_{G}(y)-2, & \text { if } x y \in E(G)\end{cases}
$$




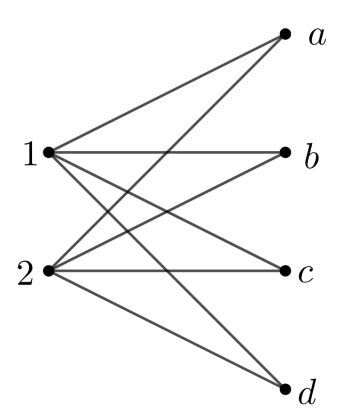

(a)

Figure 1.1: (a) Complete bipartite graph $K_{2,4}$
$\{2, d\}$

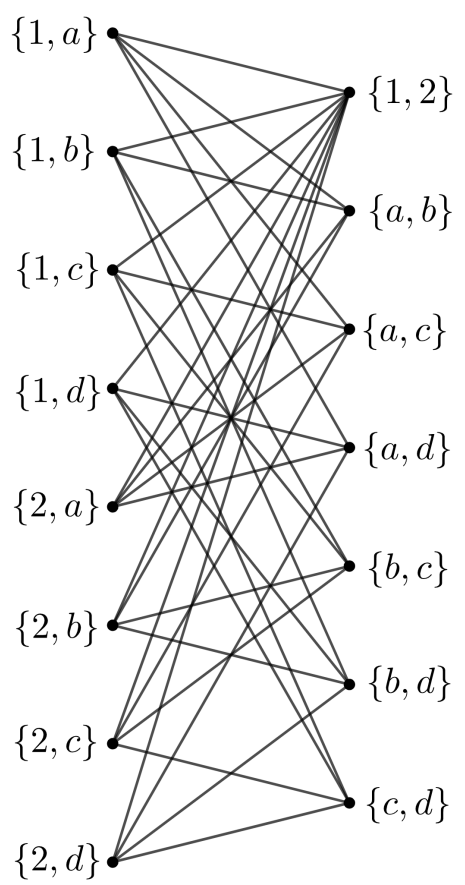

$(b)$

(b) Double vertex graph of $K_{2,4}$

Token graphs have been extensively studied especially in terms of the combinatorial parameters such as connectivity, diameter, cliques, chromatic number, Hamiltonian paths and Cartesian product (see [7]-[14] and the references therein).

The connectivity, $\kappa(G)$, of a graph $G$ is the minimum number of vertices whose removal from $G$ results in a disconnected graph or an isolated vertex. It is an important factor to determine the fault-tolerance of a network. In 1983, Harary introduced conditional connectivity as a generalization of the classical connectivity concept by imposing some conditions on the remaining graph. Let $G$ be a connected graph, and let $P$ be a given graph-theoretical property. The conditional connectivity of a graph $G$ is the size of a minimum vertex-cut $S$ of $G$ (if it exists), where $G-S$ is disconnected and every component of $G-S$ has the property $P$ [15]. Motivated by this definition, various types of conditional connectivity have been extensively studied in literature. The case when the condition is that the remaining graph does not have an isolated vertex corresponds to the super-connectivity notion.

The super-connectivity, $\kappa^{\prime}(G)$, of a graph $G$ is the size of a minimum vertex-cut $S$ such that the resulting graph $G-S$ has no isolated vertices. If such a vertex-cut exists, it is referred to as a super vertex-cut; otherwise we write $\kappa^{\prime}(G)=+\infty$. The super-connectivity has been studied for various families of graphs, including circulant graphs [16], hypercubes [17, 18], product graphs [19]-[21].

Considering the connectivity aspect of token graphs, it is known that if $G$ is a $k$-connected graph, then $F_{2}(G)$ is $(2 k-2)-$ connected, where $k \geq 3$ [3]. In 2012, Fabila-Monroy et al. [6] presented several families of graphs of order $n$ which are $t$-connected and have $k$-token graphs with connectivity exactly $k(t-k+1)$ whenever $k \leq t$. They also conjectured that $F_{k}(G)$ is at least $k(t-k+1)$-connected for all $k \leq t$. In 2018, Leaños and Trujillo-Negrete [22] proved that their conjecture is true. In [23], Leaños and Ndjatchi proved an analogous result for edge connectivity; they showed that if $G$ is $t$-edge connected for $t \geq k$, then $F_{k}(G)$ is at least $k(t-k+1)$-edge connected. Later Fabila-Monroy et al. [24] proved that if $G$ is a tree, then the connectivity of $F_{k}(G)$ is equal to the minimum degree of $F_{k}(G)$. Although the connectivity of $k$-token graphs has been studied in several papers, super-connectivity of this class has not yet been investigated. Recently, we fully determined the super-connectivity of Johnson graphs, which corresponds to a special case of $k$-token graphs [25]. More precisely, if $G$ is the complete graph on $n$ vertices, then $k$-token graph corresponds to the Johnson graph $J(n, k)$. In this paper, we continue to investigate token graphs by determining the super-connectivity of 2-token graph of complete bipartite graphs.

In the rest of the paper, a vertex $\omega$ of $F_{2}(G)$ corresponding to the 2-subset $\{x, y\} \in V\left(F_{2}(G)\right)$ will be denoted by $\omega=x y$. While constructing the paths, it is assumed that the subscripts of the vertices are taken modulo $n$ or $m$, depending on the size of the set we consider. 


\section{Main results}

Let $K_{m, n}$ be the complete bipartite graph with partition $V=A \cup B$ such that $A=\left\{x_{1}, \ldots, x_{m}\right\}$ and $B=\left\{y_{1}, \ldots, y_{n}\right\}$, where $m \leq n$. Letting $\mathscr{G}=F_{2}\left(K_{m, n}\right)$, we have a bipartite graph $\mathscr{G}$ with partition $V(\mathscr{G})=\mathscr{A} \cup \mathscr{B}$ such that

$$
\mathscr{A}=\left\{x_{i} y_{j} \in V(\mathscr{G}): x_{i} \in A \text { and } y_{j} \in B\right\} \quad \text { and } \quad \mathscr{B}=\mathscr{B}_{1} \cup \mathscr{B}_{2},
$$

where

$$
\mathscr{B}_{1}=\left\{x_{i} x_{j} \in V(\mathscr{G}): i \neq j\right\} \quad \text { and } \quad \mathscr{B}_{2}=\left\{y_{i} y_{j} \in V(\mathscr{G}): i \neq j\right\} .
$$

It is easy to see that $\delta(\mathscr{G})=\min \{2 m, 2 n, m+n-2\}$. Since $\kappa\left(K_{m, n}\right)=m$ when $m \leq n$, we know that the graph $\mathscr{G}$ is $(2 m-2)-$ connected for $n \geq m \geq 2$. We know that the connectivity of a graph is at most the minimum degree of it. Thus, we have $2 m-2 \leq \kappa(\mathscr{G}) \leq 2 m$ when $n \geq m+2$. Moreover, if $m=n$, then $\kappa(\mathscr{G})=2 m-2$ and if $m=n-1$, then $2 m-2 \leq \kappa(\mathscr{G}) \leq 2 m-1$. It is quite natural to ask whether every minimum vertex-cut of a graph $G$ corresponds to the neighbourhood of a vertex. If the answer is yes, then every vertex-cut isolates a vertex in $G$ and thus the super-connectivity of $G$ is strictly greater than the connectivity.

Both the Remark 2.1 and the explanation before it are given in [25]. Although it is easy to observe, it plays an important role in the proof of our main result.

Let $S$ be a minimum super vertex-cut $S$ of a connected graph $G$. Note that $S$ contains a vertex $v$ having at least one neighbour in the resulting graph $G-S$ for otherwise $G$ would be disconnected. Let $C$ be a component of $G-S$ and suppose that $v$ does not have a neighbour in $C$. Now consider the set $T=S-\{v\}$. Since $C$ is a component of $G-T$, it is obvious that $T$ is a vertex-cut of $G$ which does not isolate a vertex. Thus, $T$ is a super vertex-cut of $G$ and this contradicts the minimality of $S$. Hence, the remark below follows.

Remark 2.1. [25] Let $G$ be a connected graph. A minimum super vertex-cut $S$ of $G$ contains a vertex having at least a neighbour in every component of $G-S$. Moreover, if a vertex $v$ in a minimum super vertex-cut $S$ of $G$ has a neighbour in one component of $G-S$, then it has at least one neighbour in every component of $G-S$.

We now prove our main result on the super-connectivity of the double vertex graph of complete bipartite graphs.

Theorem 2.2. Let $\mathscr{G}$ be the double vertex graph of the complete bipartite graph $K_{m, n}$, where $n \geq m+2$ and $m \geq 4$. Then $\kappa^{\prime}(\mathscr{G})=3 m+n-4$.

Proof. Let $S$ be a super vertex-cut of $\mathscr{G}=F_{2}\left(K_{m, n}\right)$ where $n \geq m+2$ and $m \geq 4$. By Remark 2.1, we know that there exists a vertex, say $\omega$, in $S$ having at least a neighbour in every component of $\mathscr{G}-S$. Let $C_{1}$ and $C_{2}$ be two components of $\mathscr{G}-S$. Consider a neighbour of $\omega$ from each of the components $C_{1}$ and $C_{2}$, say $u_{1} \in C_{1}$ and $u_{2} \in C_{2}$. Since $S$ is a super vertex-cut, each component of the resulting graph $\mathscr{G}-S$ has at least two vertices. Thus, each of $u_{1}$ and $u_{2}$ has at least a neighbour in $C_{1}$ and $C_{2}$, respectively. Let $v_{1} \in C_{1}$ and $v_{2} \in C_{2}$ such that $v_{1} \in N_{\mathscr{G}}\left(u_{1}\right)$ and $v_{2} \in N_{\mathscr{G}}\left(u_{2}\right)$. Note that the intersection $v_{1} \cap u_{2}=\emptyset$, otherwise there will be an edge between the components $C_{1}$ and $C_{2}$. Similarly, $u_{1} \cap v_{2}=\emptyset$. Since $\mathscr{G}$ is a bipartite graph, $\omega$ is either in $\mathscr{A}$ or in $\mathscr{B}$.

First, we suppose that $\omega$ is in $\mathscr{A}$. Without loss of generality, let $\omega=x_{1} y_{1}$. For the vertices $u_{1}$ and $u_{2}$, there are three cases to consider:

(1) Both of $u_{1}$ and $u_{2}$ are in $\mathscr{B}_{1}$,

(2) One of them is in $\mathscr{B}_{1}$ and the other one is in $\mathscr{B}_{2}$,

(3) Both of $u_{1}$ and $u_{2}$ are in $\mathscr{B}_{2}$.

Next, we suppose that $\omega$ is in $\mathscr{B}$. Then, either $\omega \in \mathscr{B}_{1}$ or $\omega \in \mathscr{B}_{2}$. In both of these two cases, we get the same subcases for the vertices $u_{1}, v_{1} \in C_{1}$ and $u_{2}, v_{2} \in C_{2}$. Thus, it is enough to consider only one of them, say $\omega \in \mathscr{B}_{1}$. Without loss of generality, we assume that $\omega=x_{1} x_{2}$. Consider the neighbours of $\omega$ in the resulting graph $\mathscr{G}-S$, in particular $u_{1} \in C_{1}$ and $u_{2} \in C_{2}$. Due to the shared index of $u_{1}$ and $u_{2}$, there are three cases to consider:

(4) $u_{1} \cap u_{2} \subset A$,

(5) $u_{1} \cap u_{2} \subset B$,

(6) $u_{1} \cap u_{2}=\emptyset$.

Let us assume that $\omega=x_{1} y_{1}$ and consider the first three cases (1-3) given below.

Case 1. Without loss of generality, assume that $u_{1}=x_{1} x_{2}$ and $u_{2}=x_{1} x_{3}$. Since we have $v_{1} \cap u_{2}=\emptyset$ and $u_{1} \cap v_{2}=\emptyset$, we let $v_{1}=x_{2} y_{k}$ and $v_{2}=x_{3} y_{\ell}$. Without loss of generality, we assume that $k=1$. Thus, we have either $\ell=k$ or $\ell \neq k$. In the latter case we let, without loss of generality, $\ell=2$.

First we investigate the common paths that can be constructed when either $\ell=k$ or $\ell \neq k$. 
- $u_{1} \sim x_{1} y_{j} \sim u_{2}$ for all $j \in\{1, \ldots, n\}$

- $v_{1} \sim x_{2} x_{3} \sim v_{2}$

- $v_{1} \sim x_{2} x_{j} \sim y_{2} x_{j} \sim x_{3} x_{j} \sim v_{2}$ for all $j \in\{4, \ldots, m\}$

Note that if $\ell=k$, then the vertices $v_{1}$ and $v_{2}$ have common neighbours, and the additional paths that can be constructed particularly in this case are given in $(a)$. Similarly, the additional paths constructed only when $\ell=2$ are given in $(b)$.

(a) If $\ell=k$, then consider the extra paths given below:

- $v_{1} \sim y_{1} y_{j} \sim v_{2}$ for all $j \in\{2, \ldots, n\}$

- $u_{1} \sim x_{2} y_{j} \sim y_{j} y_{j+1} \sim x_{3} y_{j} \sim u_{2}$ for all $j \in\{2, \ldots, n\}$

When $j=n$, use the vertex $y_{j} y_{j+2}$ instead of $y_{j} y_{j+1}$ since $y_{1} y_{n}$ is used already.

(b) If $\ell \neq k$ (note that $\ell$ is assumed to be 2 above), then consider the extra paths given below:

- $u_{1} \sim x_{2} y_{2} \sim y_{2} y_{3} \sim v_{2}$ and $u_{1} \sim x_{2} y_{3} \sim y_{3} y_{4} \sim x_{3} y_{3} \sim u_{2}$

- $v_{1} \sim y_{1} y_{2} \sim v_{2}$ and $v_{1} \sim y_{1} y_{3} \sim x_{3} y_{1} \sim u_{2}$

- $v_{1} \sim y_{1} y_{j} \sim x_{3} y_{j} \sim u_{2}$ for all $j \in\{4, \ldots, n\}$

- $u_{1} \sim x_{2} y_{j} \sim y_{2} y_{j} \sim v_{2}$ for all $j \in\{4, \ldots, n\}$

Thus, in both cases, we have constructed $3 n+m-4$ internally disjoint paths.

Case 2. Without loss of generality, we let $u_{1}=x_{1} x_{2}$ and $u_{2}=y_{1} y_{2}$. Since we have $v_{1} \cap u_{2}=\emptyset$ and $u_{1} \cap v_{2}=\emptyset$, we assume that $v_{1}=x_{1} y_{3}$ and $v_{2}=x_{3} y_{1}$. Consider the following paths:

- $u_{1} \sim x_{1} y_{i} \sim y_{2} y_{i} \sim x_{i} y_{2} \sim u_{2}$ for all $i \in\{4, \ldots, m\}$

- $u_{1} \sim x_{2} y_{j} \sim y_{1} y_{j} \sim v_{2}$ for all $j \in\{4, \ldots, n\}$

- $v_{1} \sim x_{1} x_{i} \sim x_{i} y_{1} \sim u_{2}$ for all $i \in\{4, \ldots, m\}$

- $v_{1} \sim y_{3} y_{i} \sim x_{i} y_{3} \sim x_{3} x_{i} \sim v_{2}$ for all $i \in\{4, \ldots, m\}$

- $u_{1} \sim x_{2} y_{3} \sim x_{2} x_{3} \sim v_{2}$ and $v_{1} \sim y_{2} y_{3} \sim x_{3} y_{2} \sim u_{2}$

- $u_{1} \sim a \sim u_{2}$ for each $a \in\left\{x_{1} y_{1}, x_{1} y_{2}, x_{2} y_{1}, x_{2} y_{2}\right\}$

- $v_{1} \sim x_{1} x_{3} \sim v_{2}$ and $v_{1} \sim y_{1} y_{3} \sim v_{2}$

Thus, we have constructed $3 m+n-4$ internally disjoint paths.

Case 3. Without loss of generality, we let $u_{1}=y_{1} y_{2}$ and $u_{2}=y_{1} y_{3}$. Since we have $v_{1} \cap u_{2}=\emptyset$ and $u_{1} \cap v_{2}=\emptyset$, we let $v_{1}=x_{k} y_{2}$ and $v_{2}=x_{\ell} y_{3}$. Without loss of generality, we assume that $k=1$. Thus, we have either have $\ell=k$ or $\ell \neq k$. In the latter case we let, without loss of generality, $\ell=2$.

First we investigate the common paths that can be constructed when either $\ell=k$ or $\ell \neq k$

- $u_{1} \sim x_{i} y_{1} \sim u_{2}$ for all $i \in\{1, \ldots, m\}$

- $v_{1} \sim y_{2} y_{3} \sim v_{2}$

- $v_{1} \sim y_{2} y_{i} \sim x_{1} y_{i} \sim y_{3} y_{i} \sim v_{2}$ for all $i \in\{4, \ldots, n\}$

Note that if $\ell=k$, then the vertices $v_{1}$ and $v_{2}$ have common neighbours, and the additional paths that can be constructed particularly in this case are given in $(a)$. Similarly, the additional paths constructed only when $\ell=2$ are given in $(b)$.

(a) If $\ell=k$, then consider the extra paths given below:

- $v_{1} \sim x_{1} x_{i} \sim v_{2}$ for all $i \in\{2, \ldots, m\}$

- $u_{1} \sim x_{i} y_{2} \sim x_{i} x_{i+1} \sim x_{i} y_{3} \sim u_{2}$ for all $i \in\{2, \ldots, m\}$.

When $i=m$, use the vertex $x_{i} x_{i+2}$ instead of $x_{i} x_{i+1}$ since $x_{1} x_{m}$ is used already.

(b) If $\ell \neq k$ ( $\ell$ is assumed to be 2 above), then consider the extra paths given below:

- $u_{1} \sim x_{2} y_{2} \sim x_{2} x_{3} \sim v_{2}$ and $v_{1} \sim x_{1} x_{3} \sim x_{1} y_{3} \sim u_{2}$

- $u_{1} \sim x_{3} y_{2} \sim x_{3} x_{4} \sim x_{3} y_{3} \sim u_{2}$

- $u_{1} \sim x_{4} y_{2} \sim x_{2} x_{4} \sim v_{2}$ and $v_{1} \sim x_{1} x_{4} \sim x_{4} y_{3} \sim u_{2}$

- $v_{1} \sim x_{1} x_{2} \sim v_{2}$

- $u_{1} \sim x_{i} y_{2} \sim x_{i-1} x_{i} \sim x_{i} y_{3} \sim u_{2}$ for all $i \in\{5, \ldots, m\}$ 
- $v_{1} \sim x_{1} x_{i} \sim x_{i} y_{4} \sim x_{2} x_{i} \sim v_{2}$ for all $i \in\{5, \ldots, m\}$

Thus, in both cases, we have constructed $3 m+n-4$ internally disjoint paths.

Now we assume that $\omega=x_{1} x_{2}$ in order to consider the latter three cases (4-6) given below.

Case 4. Let $u_{1} \cap u_{2} \subset A$, say $u_{1} \cap u_{2}=\left\{x_{1}\right\}$. Since both of $u_{1}, u_{2} \in \mathscr{A}$, without of loss of generality, we assume that $u_{1}=x_{1} y_{1}$ and $u_{2}=x_{1} y_{2}$. Since we have $v_{1} \cap u_{2}=\emptyset$ and $v_{2} \cap u_{1}=\emptyset$, we have either $\left|v_{1} \cap v_{2}\right|=1$ or $\left|v_{1} \cap v_{2}\right|=0$. Let $v_{1}=y_{1} y_{k}$ and $v_{2}=y_{2} y_{\ell}$. Note that $k, \ell \notin\{1,2\}$. Thus, without loss of generality, we let $k=3$.

(a) If $\ell=k$, then the paths here can be constructed similarly as in Case $3(a)$, such that the vertices $\left\{x_{1}, y_{1}, y_{2}, y_{3}\right\}$ of this case correspond to the vertices $\left\{x_{1}, y_{2}, y_{3}, y_{1}\right\}$ of Case $3(a)$, respectively.

(b) If $\ell \neq k$, then we have $\ell \notin\{1,2,3\}$. Thus, without loss of generality, we let $\ell=4$.

Consider the following paths:

- $u_{1} \sim x_{1} x_{i} \sim u_{2}$ for all $i \in\{2, \ldots, m\}$

- $u_{1} \sim y_{1} y_{2} \sim u_{2}$

- $u_{1} \sim y_{1} y_{4} \sim x_{1} y_{4} \sim v_{2}$

- $v_{1} \sim x_{1} y_{3} \sim y_{2} y_{3} \sim u_{2}$

- $u_{1} \sim y_{1} y_{i} \sim x_{1} y_{i} \sim y_{2} y_{i} \sim u_{2}$ for all $i \in\{5, \ldots, n\}$

- $v_{1} \sim x_{i} y_{1} \sim x_{i} x_{i+1} \sim x_{i} y_{2} \sim v_{2}$ for all $i \in\{2, \ldots, m\}$

When $i=m$, use the vertex $x_{i} x_{i+2}$ instead of $x_{i} x_{i+1}$ since $x_{1} x_{m}$ is used already.

- $v_{1} \sim x_{2} y_{3} \sim y_{3} y_{4} \sim x_{2} y_{4} \sim v_{2}$

- $v_{1} \sim x_{i} y_{3} \sim y_{3} y_{i+2} \sim x_{2} y_{i+2} \sim y_{4} y_{i+2} \sim x_{i} y_{4} \sim v_{2}$ for all $i \in\{3, \ldots, m\}$

Thus, in both of the cases, we have constructed $3 m+n-4$ internally disjoint paths.

Case 5. Let $u_{1} \cap u_{2} \subset B$, say $u_{1} \cap u_{2}=\left\{y_{1}\right\}$. Since both of $u_{1}, u_{2} \in \mathscr{A}$, without of loss of generality, we assume that $u_{1}=x_{1} y_{1}$ and $u_{2}=x_{2} y_{1}$. Since $v_{1} \cap u_{2}=\emptyset$ and $v_{2} \cap u_{1}=\emptyset$, we have either $\left|v_{1} \cap v_{2}\right|=1$ or $\left|v_{1} \cap v_{2}\right|=0$. Let $v_{1}=x_{1} x_{k}$ and $v_{2}=x_{2} x_{\ell}$. Note that $k, \ell \notin\{1,2\}$. Thus, without loss of generality, we let $k=3$.

(a) If $\ell=k$ then the paths here can be constructed similarly as in Case 1(a), such that the vertices $\left\{y_{1}, x_{1}, x_{2}, x_{3}\right\}$ of this case correspond to the vertices $\left\{y_{1}, x_{2}, x_{3}, x_{1}\right\}$ of Case $1(a)$, respectively.

(b) If $\ell \neq k$, then we have $\ell \notin\{1,2,3\}$. Thus, without loss of generality, we let $\ell=4$.

Consider the following paths:

- $u_{1} \sim x_{1} x_{2} \sim u_{2}$

- $u_{1} \sim y_{1} y_{i} \sim u_{2}$ for all $i \in\{2, \ldots, n\}$

- $u_{1} \sim x_{1} x_{4} \sim x_{4} y_{m} \sim v_{2}$ and $v_{1} \sim x_{3} y_{m} \sim x_{2} x_{3} \sim u_{2}$

- $u_{1} \sim x_{1} x_{i} \sim x_{i} y_{1} \sim x_{2} x_{i} \sim u_{2}$ for all $i \in\{5, \ldots, m\}$

- $v_{1} \sim x_{3} y_{1} \sim x_{3} x_{4} \sim x_{4} y_{1} \sim v_{2}$

- $v_{1} \sim x_{1} y_{i} \sim y_{i} y_{i+1} \sim x_{2} y_{i} \sim v_{2}$ for all $i \in\{2, \ldots, n\}$

When $i=n$, use the vertex $y_{i} y_{i+2}$ instead of $y_{i} y_{i+1}$ since $y_{1} y_{n}$ is used already.

- $v_{1} \sim x_{3} y_{i} \sim y_{i} y_{i+2} \sim x_{4} y_{i} \sim v_{2}$ for all $i \in\{2, \ldots, n-1\}$

When $i=n-1$, use the vertex $y_{i} y_{i+3}$ instead of $y_{i} y_{i+2}$ since $y_{1} y_{n-1}$ is used already.

Thus, in both of the cases, we have constructed $3 n+m-4$ internally disjoint paths.

Case 6. Let $u_{1} \cap u_{2}=\emptyset$. Since both of $u_{1}, u_{2} \in \mathscr{A}$, the vertices $v_{1}$ and $v_{2}$ are in $\mathscr{B}$. There are three subcases to consider: $(a)$ Both of $v_{1}, v_{2}$ are in $\mathscr{B}_{1},(b)$ One of $v_{1}, v_{2}$ is in $\mathscr{B}_{1}$ and the other one is in $\mathscr{B}_{2},(c)$ Both of $v_{1}, v_{2}$ are in $\mathscr{B}_{2}$.

First, without loss of generality, we let $u_{1}=x_{1} y_{1}$ and $u_{2}=x_{2} y_{2}$.

(a) Assume that $v_{1}, v_{2} \in \mathscr{B}_{1}$. Since $v_{1} \cap u_{2}=\emptyset$ and $v_{2} \cap u_{1}=\emptyset$, we have either $\left|v_{1} \cap v_{2}\right|=1$ or $\left|v_{1} \cap v_{2}\right|=0$. Let $v_{1}=x_{1} x_{k}$ and $v_{2}=x_{2} x_{\ell}$. Note that we have $k, \ell \notin\{1,2\}$. Thus, without loss of generality, we let $k=3$.

(i) If $\ell=k$, then the paths here can be constructed similarly as in Case $1(b)$, such that the vertices $\left\{x_{1}, x_{2}, x_{3}, y_{1}, y_{2}\right\}$ of this case correspond to the vertices $\left\{x_{2}, x_{3}, x_{1}, y_{1}, y_{2}\right\}$ of Case $1(b)$, respectively.

(ii) If $\ell \neq k$, then we have $\ell \notin\{1,2,3\}$. Thus, without loss of generality, let $\ell=4$.

Consider the following paths: 
- $u_{1} \sim x_{1} x_{2} \sim u_{2}$ and $u_{1} \sim y_{1} y_{2} \sim u_{2}$

- $u_{1} \sim x_{1} x_{4} \sim x_{4} y_{1} \sim v_{2}$ and $v_{1} \sim x_{3} y_{1} \sim x_{2} x_{3} \sim u_{2}$

- $u_{1} \sim x_{1} x_{i} \sim x_{i} y_{1} \sim x_{2} x_{i} \sim u_{2}$ for all $i \in\{5, \ldots, m\}$

- $u_{1} \sim y_{1} y_{3} \sim x_{2} y_{1} \sim v_{2}$ and $v_{1} \sim x_{1} y_{2} \sim y_{2} y_{3} \sim u_{2}$

- $u_{1} \sim y_{1} y_{i} \sim x_{4} y_{i} \sim v_{2}$ for all $i \in\{4, \ldots, n\}$

- $v_{1} \sim x_{3} y_{i} \sim y_{2} y_{i} \sim u_{2}$ for all $i \in\{4, \ldots, n\}$

- $v_{1} \sim x_{1} y_{i} \sim y_{i} y_{i+1} \sim x_{2} y_{i} \sim v_{2}$ for all $i \in\{3, \ldots, n\}$

When $i=n$, use the vertex $y_{i} y_{i+3}$ instead of $y_{i} y_{i+1}$ since $y_{1} y_{n}$ is used already.

- $v_{1} \sim x_{3} y_{2} \sim x_{3} x_{4} \sim x_{4} y_{2} \sim v_{2}$

- $v_{1} \sim x_{3} y_{3} \sim y_{3} y_{5} \sim x_{4} y_{3} \sim v_{2}$

Thus, in both of the cases, we have constructed $3 n+m-4$ internally disjoint paths.

(b) Assume that $v_{1} \in \mathscr{B}_{1}$ and $v_{2} \in \mathscr{B}_{2}$. Since $v_{1} \cap u_{2}=\emptyset$ and $v_{2} \cap u_{1}=\emptyset$, we let $v_{1}=x_{1} x_{3}$ and $v_{2}=y_{2} y_{3}$. The paths here can be constructed similarly as in Case 2, such that the vertices $\left\{x_{1}, x_{2}, x_{3}, y_{1}, y_{2}, y_{3}\right\}$ of this case correspond to the vertices $\left\{x_{1}, x_{3}, x_{2}, y_{3}, y_{1}, y_{2}\right\}$ of Case 2 , respectively. Thus, we can construct $3 m+n-4$ internally disjoint paths.

(c) Assume that $v_{1}, v_{2} \in \mathscr{B}_{2}$. Since $v_{1} \cap u_{2}=\emptyset$ and $v_{2} \cap u_{1}=\emptyset$, we have either $\left|v_{1} \cap v_{2}\right|=1$ or $\left|v_{1} \cap v_{2}\right|=0$. Let $v_{1}=y_{1} y_{k}$. Note that $k \notin\{1,2\}$. Thus, without loss of generality, we let $k=3$.

Since $v_{2} \in \mathscr{B}_{2}$ by the assumption, we have $v_{2}=y_{2} y_{\ell}$ such that $\ell \notin\{1,2\}$. Then we have either $\ell=k$ or $\ell \neq k$.

(i) If $\ell=k$, then the paths here can be constructed similarly as in Case 3(b), such that the vertices $\left\{x_{1}, x_{2}, y_{1}, y_{2}, y_{3}\right\}$ of this case correspond to the vertices $\left\{x_{1}, x_{2}, y_{2}, y_{3}, y_{1}\right\}$ of Case $3(b)$, respectively.

(ii) If $\ell \neq k$, then we have $\ell \notin\{1,2,3\}$. Thus, without loss of generality, we let $v_{2}=y_{2} y_{4}$.

Consider the following paths:

- $u_{1} \sim x_{1} x_{2} \sim u_{2}$ and $u_{1} \sim y_{1} y_{2} \sim u_{2}$

- $u_{1} \sim x_{1} x_{3} \sim x_{1} y_{2} \sim v_{2}$ and $v_{1} \sim x_{2} y_{1} \sim x_{2} x_{3} \sim u_{2}$

- $u_{1} \sim x_{1} x_{i} \sim x_{i} y_{2} \sim v_{2}$ for all $i \in\{4, \ldots, m\}$

- $u_{1} \sim y_{1} y_{4} \sim x_{1} y_{4} \sim v_{2}$ and $v_{1} \sim x_{1} y_{3} \sim y_{2} y_{3} \sim u_{2}$

- $v_{1} \sim x_{2} y_{3} \sim y_{3} y_{4} \sim x_{2} y_{4} \sim v_{2}$ and $v_{1} \sim x_{3} y_{1} \sim x_{3} x_{4} \sim x_{3} y_{2} \sim v_{2}$

- $u_{1} \sim y_{1} y_{i} \sim x_{1} y_{i} \sim y_{2} y_{i} \sim u_{2}$ for all $i \in\{5, \ldots, n\}$

- $v_{1} \sim x_{i} y_{3} \sim y_{3} y_{i+2} \sim x_{2} y_{i+2} \sim y_{4} y_{i+2} \sim x_{i} y_{4} \sim v_{2}$ for all $i \in\{3, \ldots, m\}$

- $v_{1} \sim x_{i} y_{1} \sim x_{2} x_{i} \sim u_{2}$ for all $i \in\{4, \ldots, m\}$

Thus, in both cases, we have constructed $3 m+n-4$ internally disjoint paths.

In each of the six cases above, we presented either $3 m+n-4$ or $3 n+m-4$ internally disjoint paths between $C_{1}$ and $C_{2}$. Since $m \leq n-2$ by the assumption, this implies that there exist at least $3 m+n-4$ internally disjoint paths between $C_{1}$ and $C_{2}$. Thus, $\kappa^{\prime}(\mathscr{G}) \geq 3 m+n-4$.

On the other hand, consider two adjacent vertices $\alpha$ and $\beta$ of $\mathscr{G}$ such that $\alpha \in \mathscr{A}$ and $\beta \in \mathscr{B}$. Let $S=\left(N_{\mathscr{G}}(\alpha) \cup N_{\mathscr{G}}(\beta)\right)-$ $\{\alpha, \beta\}$. It is easy to see that the set $S$ disconnects the graph without isolating a vertex, that is, $S$ is a super-vertex cut of $\mathscr{G}$. Hence, we get $\kappa^{\prime}(\mathscr{G}) \leq|S|=3 m+n-4$ and this finishes the proof.

\section{Conclusion}

In our main result, it is proved that the super-connectivity of the double vertex graph of complete bipartite graph $K_{m, n}$ is equal to $3 m+n-4$ where $m \geq 4$ and $n \geq m+2$. This result also implies that the double vertex graph of complete bipartite graph $F_{2}\left(K_{m, n}\right)$ is super-connected, i.e., each minimum vertex-cut of $F_{2}\left(K_{m, n}\right)$ isolates a vertex. It would be interesting to determine the super-connectivity of $k$-token graphs for larger graph classes. Note that the well studied Johnson graph $J(n, k)$ is a special case of $k$-token graphs, where $G$ is the complete graph $K_{n}$. In [25], we fully determined the super-connectivity of $J(n, k)$. Thus, the results given in [25] might be generalized by a possible study on $k$-token graphs of larger graph classes. 


\section{Acknowledgements}

The authors would like to express their sincere thanks to the editor and the anonymous reviewers for their helpful comments and suggestions.

\section{Funding}

There is no funding for this work.

\section{Availability of data and materials}

Not applicable.

\section{Competing interests}

The authors declare that they have no competing interests.

\section{Author's contributions}

All authors contributed equally to the writing of this paper. All authors read and approved the final manuscript.

\section{References}

[1] Y. Alavi, M. Behzad, P. Erdos, D. R. Lick, Double vertex graphs, J. Comb. Inf. Syst. Sci., 16(1) (1991), 37-50.

[2] Y. Alavi, M. Behzad, J. E. Simpson, Planarity of Double Vertex Graphs, Graph theory, combinatorics, algorithms, and applications (San Francisco, CA) (1991), 472-485.

[3] Y. Alavi, D. R. Lick, J. Liu, Survey of double vertex graphs, Graphs Combin., 18(4) (2002), 709-715.

[4] T. Rudolph, Constructing physically intuitive graph invariants, (2002), arXiv:quant-ph/0206068.

[5] K. Audenaert, C. Godsil, G. Royle, T. Rudolph, Symmetric squares of graphs, J. Combin. Theory Ser. B, 97(1) (2007), 74-90.

[6] R. Fabila-Monroy, D. Flores-Peñaloza, C. Huemer, F. Hurtado, J. Urrutia, D. R. Wood, Token graphs, Graphs Combin., 28(3) (2012), 365-380.

[7] L. Adame, L. M. Rivera, A. L. Trujillo-Negrete, Hamiltonicity of the double vertex graph and the complete double vertex graph of some join graphs, (2020), arXiv:2007.00115 [math.CO]

[8] L. Adame, L. M. Rivera, A. L. Trujillo-Negrete, Hamiltonicity of token graphs of some join graphs, Symmetry, 13(6) (2021), 1076.

[9] H. de Alba, W. Carballosa, J. Leaños, L. M. Rivera, Independence and matching numbers of some token graphs, Australas. J. Combin., 76 (2020),

[10] J. Deepalakshmi, G. Marimuthu, Characterization of token graphs, J. Eng. Technol., 6 (2017), 310-317.

[11] J. Deepalakshmi, G. Marimuthu, A. Somasundaram, S. Arumugam, On the 2-token graph of a graph, AKCE Int. J. Graphs Comb., 17(1) (2019), $265-268$

[12] P. Jiménez-Sepúlveda, L. M. Rivera, Independence numbers of some double vertex graphs and pair graphs, (2018), arXiv:1810.06354 [math.CO].

[13] S. S. Kumar, R. Sundareswaran, M. Sundarakannan, On Zagreb indices of double vertex graphs, TWMS J. Appl. Eng. Math., 10(4) (2020), 1096-1104.

[14] J. G. Soto, J. Leaños, L. M. Ríos-Castro, L. M. Rivera, The packing number of the double vertex graph of the path graph, Discrete Appl. Math., 247 (2018), 327-340.

[15] F. Harary, Conditional connectivity, Networks, 13(3) (1983), 347-357.

[16] F. Boesch, R. Tindell, Circulants and their connectivities, J. Graph Theory, 8(4) (1984), 487-499.

[17] W. Yang, J. Meng, Extraconnectivity of hypercubes, Appl. Math. Lett., 22(6) (2009), 887-891.

[18] W. Yang, J. Meng, Extraconnectivity of hypercubes (II), Australas. J. Comb., 47 (2010), 189-196.

[19] G. B. Ekinci, A. Kurlangiç, Super connectivity of Kronecker product of complete bipartite graphs and complete graphs, Discrete Math., 339(7) (2016), 1950-1953.

[20] L. Guo, C. Qin, X. Guo, Super connectivity of Kronecker products of graphs, Inform. Process. Lett., 110 (16) (2010), 659-661.

[21] M. Lü, C. Wu, G.-L. Chen, C. Lv, On super connectivity of Cartesian product graphs, Networks, 52(2) (2008), 78-87.

[22] J. Leaños, A. L. Trujillo-Negrete, The connectivity of token graphs, Graphs Combin., 34(4) (2018), 777-790.

[23] J. Leaños, C. Ndjatchi, The edge-connectivity of token graphs, Graphs Combin., 37(3) (2021), 1013-1023.

[24] R. Fabila-Monroy, J. Leaños, A. L. Trujillo-Negrete, On the connectivity of token graphs of trees, (2020), arXiv:2004.14526 [math.CO].

[25] G. B. Ekinci, J. B. Gauci, The super-connectivity of Johnson graphs, Discrete Math. Theor. Comput. Sci., 22(1) (2020). 